\title{
Ueber die sogenannte diphtheroïde Form des venerischen Gesehwürs auf dem Cervix uteri.
}

\author{
Von
}

Dr. C. Rasch,

erstem Assistenten an der Klinik für Hautkrankheiten der Universität Kopenhagen.

Das venerische Geschwür (der weiche Schanker) auf dem Halse der Gebärmutter gehört zu den ziemlich selten rorkommenden Affectionen. Da dasselbe auch morphologische Eigenthümlichkeiten darbietet, welche von den gewöhnlichen Typen dieses Schankers stark abweichen, und welche Veranlassung zu diagnostischen Irrthümern geben können, habe ich es der Mühe werth erachtet, folgende drei Fälle, welche ich in der letzten Zeit wahrzunehmen Gelegenheit gehabt habe, mitzutheilen.

1. Fall. 36jährige Frau, welche am 22. Februar 1896 in die dermatologische Klinik des Communehospitals gebracht wurde, deren Chef, Prof. Haslund ich für die Erlaubniss, das Journal benutzen zu dürfen, hiermit danke. Der Mann der Patientin wurde drei Wochen vorher im Hospital an einem venerischen Geschwür auf dem Frenulum, dem ein beiderseitiger Bubo folgte, behandelt. Die Pat. hatte vor 2 Monaten zum vierten Mal geboren. Das Kind, dem nichts fehlte, wurde gleichzeitig des Stillens wegen aufgenommen. Pat. theilte mit, dass sie ungefähr seit einem Monat einen eiterigen Ausfluss aus der Scheide gehabt habe. Irgend ein Geschwür an den äusseren Geschlechtstheilen habe sie indessen nicht wahrgenommen. Ein solehes war jedoch wahrscheinlich vorhanden gewesen, da sie 3 Wochen lang eine schmerzhafte Drüsengeschwulst in der linken Leistenbenge gehabt hatte. Bei der Untersuchung fand sich unbodeutender Ausfluss aus der Harnröhre und reichlicher eiteriger Ausfluss aus der Scheide. Auf dem Halse der Gebärmutter um das Orificium, 
welches an den Seiten eingerissen war, fand sich auf der etwas evertirten: Schleimhaut eine Affection, welche sich als gruppenweise auftretende, confluirende, papulöse Hervorragungen mit fein granulöser Oberfläche von gelber Farbe darstelite. Jede einzelne Hervorragung war von einer Grösse, welche zwischen Erbse und Bohne varirte. Bei der geringsten Berührung zeigte sich starke Blutung. Der Rand war gebuchtet, polycyklisch. Die Affection war nach anssen von der blassrothen Schleimbaut auf dem Cervix scharf begrenzt, schien sich aber nach innen bis in den Cervicalcanal zu erstrecken. Keine Ulcerationsprocesse an den äusseren Geschlechtstheilen. Während der Behandlung mit Alaundouche, TanninGlycerin-Tampon in der Scheide und späterer Wasserdouche wuchs die geschwulstartige Masse auf dem Collum gleichmässig und beständig im Laufe der folgenden 16 Tage. Da man wegen des geschwulstartigen Charakters der Affection nicht an die Möglichkeit eines venerischen Geschwürs glaubte und sehr geneigt war, ein beginnendes Epitheliom anzunehmen, wurde ein Keil zu mikroskopischer Untersuchung ausgeschnitten. Diese Untersuchung zeigte indessen, dass nur eine diffuse und bedeutende Rundzelleninfiltration ohne Spur von epithelialer Neubildung ') vorhanden war. Am 17. März wurde die Behandlung geändert, indem man mit täglichem Einpudern mit Jodoform und trocknem Wattetampom in die Scheide begann. Am 21. März lronnte man notiren: Nach 3tägiger Behandlung mit Jodoform ist die Affection auf dem Cervix flacher und hat an Grösse bedeutend abgenommen. Der gelbe pseadomembranöse Belag ist verschwunden, und die Farbe ist roth geworden. Da der weiche Schanker die einzige Erkrankung ist, bei welcher Jodoform eine so hervorragende, fast specifische Wirkung kat, so war so gut wie bewiesen, dass die Affection auf dem Collum ein Schanker sein müsste. Um zu noch grössererGewissheit zu kommen, wurde eine Inoculation auf dem Unterleib gemacht, welche jedoch erfolglos war, vielleicht weil das Gift durch die angewandte Jodoformbehandlung schon geschwächt worden war, oder weil man vielleicht statt des von dem. Schanker abgesonderten Eiters. etwas von dem reichlichen Secret aus dem Cervicalcanal, welches das Collum überschwemmte, genommen hatte. Indessen war uns der Zufall günstig, da ein am 24. März durch Autoinoculation entstandenes typisches venerisches Geschwür auf der Uretralpapille von länglichrunder Form, $1 \mathrm{Cm}$. lang und 3-4 Mm. breit mit scharfen Rändern und. graugelbem, anhaftendem Belag gefunden wurde.

Am 1. April war die beschriebene Affection auf der Gebärmutter vollständig verschwunden, dagegen blieb bis zum Tage der Entlassung eine rothe, leicht granulöse kreisförmige Erosion um das Orificium, aus welcher eine reichliche Absonderung eiterigen Schleims (eine einmalige

1) Die Untersuchung auf dem Ducrey'schen Bacillus ergab ein negatives Resultat. Das Präparat war aber 8 Tage in Alkohol aufbewahrt gewesen und unter diesen Umständen gelingt es nie, diese Mikroben. zu färben. (Mündliche Mittheilung von Dr. Krefting in Christiania.) 
Untersuchung zeigte keine Gonococcen) stattfand. Am 23. April war das Geschwür in der Harnröhre geheilt. Während des ganzen Aufenthaltes im Hospital hatte man dann und wann einen eiterigen Ausfluss aus der Harnröhre, welcher bei der wiederboiten Untersuchung keine Gonococcen zeigte, beobachtet. Die Drüsenentzündung in der Regio inguinalis wurde unter der Behandlung mit Eis und Bleiplatte im Laufe von 3 Wochen resorbirt. Am 30. April konnte Pat. entlassen werden, ohne dass sich bei ihr oder bei dem Kinde, welches jetzt über 4 Monate alt war, Symptome von Syphilis gezeigt hätten.

Dass die beschriebene Affection auf dem Collum ein venerisches Geschwür gewesen ist, dafür sprechen also folgende Umstände: 1. Venerisches Gesehwür bei der inficirenden Person, 2. Autoinoculation eines venerischen Geschwürs bei der Patientin selbst, 3. Heilung durch Jodoform.

2. Fall. Pat., eine 30jährige unverheiratete Arbeiterin, welche vor $31 / 2$ Jahren geboren hatte, und welche früher nicht venerisch inficirt gewesen war, gab an, 2\% Monate vor der Aufnahme (am 16. Juni) angesteckt worden zu sein. Hier wurden eine grosse Menge genitaler und perigenitaler, folliculärer Schanker und auf dem Halse der Gebärmutter mit dem Orificium als Centrum eine 2 Mark grosse, über die übrige Schleimhaut etwas hervorspringende, leicht blutende Geschwürfiäche, mit graugelbern, anhaftendem Belag gefunden. Die Inoculation von dieser Fläche auf dem Unterleib ergab einen typischen weichen Schanker. Nach 3tägiger Jodoformbehandlung wurde der graugelbe Belag abgestossen, und die Oberfläche wurde hellroth und granulös. Der Ausfluss aus dem Collum war unbedeutend, aber mit Eiter vermischt, und Pat. hatte Ausfluss ans einer paraurethralen Krypte zugleich mit jener diffusen, zähen, periurethralen Geschwulst, welche man gewöhnlich bei Frauen findet, welche einen chronischen Harnröhrentripper haben oder gehabt haben. Es zeigten sich keine Symptome von Syphilis, nur eine recht bedeutende Infiltration in einigen der geheilten Follieulitiden.

3. F all. 43jährige Frau, aufgenommen am 23. Juli, am 21. A.ug. entlassen. Vor 7 Jahren ist sie vom Manne mit Syphilis inficirt worden. Keine Recidive in den letzten drei Jahren. Ihr jetziges Leiden besteht seit 14 Tagen. (Ansteckung vor 3 Wochen), Auf den äusseren Geschlechtstheilen und auf den perigenitalen Hatpartien finden sich 13 venerische Geschwüre typischer Art und von verschiedener Grösse. (Inoculation + .) Auf der centralen Partie des Collum Uteri drei hervorragende, leicht blutende Plaques von grauröthlicher-graugelblicher Farbe und ein flaches erbsengrosses Geschwür. Reichlicher eitriger Ausfluss aus dem Cervicalcanale. Inoculation auf dem Unterleibe von den bypertrophischen Plaques gibt ein negatives Resultat, während eine von dem flachen Geschwür gemachte Inoculation positives Resultat gibt. 29. Juli 2 neue Geschwüre auf dem linken Schenkel (Autoinoculation). 21. Aug: Unter Behandlung mit warmen Bädern, Scheidenausspülungen, A etzungen mit Carbolsäure und Jodoformverband sind jetzt alle neunzehn Geschwäre vernarbt und die Patientin wird als 
gebeilt entlassen. Doch besteht natürlich noch die chronische Endometritis Wäbrend des Hospitalsanfenthaltes ist kein Zeichen von Syphilis wahrgenommen worden.

Wenn schon die renerischen Geschwüre auf dem Cervix überhaupt sehr selten sind, so scheint die beschriebene diphtheroide Form den meisten, selbst den Syphilidologen vom Fach unbekannt zu sein, obgleich dieselbe, wie wir später sehen werden, wahrscheinlich die häufigste ist. In den allermeisten Hand- und Lebrbiichern findet man nichts darüber, ja selbst in Lang's Specialarbeit (Das venerische Geschwür, Wiesbaden 1887) findet man mit keinem Worte erwähnt, dass das venerische Geschwür auf dem Collum zuweilen Eigenthümlichkeiten, wie in den oben beschriebenen Fällen, ${ }^{1}$ ) darbietet. Trotzdem ist diese klinische Form schon von dem berühmten Gynäkologen B e rn u tz in einer Mittheilung an die Soc. méd. des hôp. in Paris 1853 unter dem Namen "Chancre diphthéritique" beschrieben (cf. die unten citirte Abhandlung von $\mathrm{Schwartz}$ ). B. unterschied dieselbe indessen nicht ätiologisch von dem syphilitischen Schanker. Der erste, welcher dies that, war A. Guérin (1864) in seinen "Maladies des organes génitaux externes de la femme". Desprès (1870) warf die verschiedenen Formen wieder zusammen. 1872 erwähnt Fournier mit A. Guérin als Gewährsmann (in dem Artikel Chancre in Jaccoud's Dictionnaire), dass ausser den gewöhnlichen multiplen, tiefen, scharfgeränderten Geschwüren auf dem Halse der Gebärmutter eine Form vorkommen könne, welche als "une sorte de plaque jaunâtre ou d'un blanc grisâtre, assez analogue à une fausse membrane diphthéritique, et taisant un relief plus ou moins considérable an dessus de la muqueuse" beschrieben wird. Ein Jahr nachher 1873 machte Ch. Schwartz das venerische Geschwür und den syphilitischen Schanker auf dem Collum uteri zum Gegenstand einer Dissertation (Étude sur les chancres du col utérin. Paris 1873, Delahaye). Seine Arbeit ist ein Ausdruck für Fournier's Anschauungen und Lehren. F's. klinische Vor-

1) Dasselbe giilt von den gynäcologischen Handbüchern. Selbst in einer so neuen Arbeit wie Winter's Lehrbuch der gynäkologischen Diagnostik. Leipzig 1896, ist diese Form nicht erwähnt. Die Beschreibung des venerischen Geschwürs auf dem Cervix ist auf die übliche Beschreibung dieser Schanker, wie sie auf der Haut vorkommen, beschränkt. 
lesungen im Anfang der 70ger Jahre machte der Verwirrung und der Verwechslung der rerschiedenen Krankheiten, welche früher so allgemein gewesen war, ein Ende. F. hat später selbst (Lecons sur la syphilis chez la femme, 2 édit. 1881) bei Besprechung der Differentialdiagnose zwischen dem syphilitischen Schanker und dem venerischen Geschwür auf dem Collum eine kurz gefasste Darstellung der verschiedenen Formen, unter denen das venerische Geschwür sich auf dem Collum zeigen kann, gegeben. Im selben Jahre (1881) hat Molènes in den "Annales du dermatologie" unter dem Titel: "Contribution à l'étude de chancre non infectant du col de l'utérus (variété diphthéroide) folgende 2 Fälle von ganz derselben Art wie unser erster Fall beschrieben.

1. 21jähriges Mädehen; 2 Geburten, wonach Leukorrhoe. Bei der Untersuchung fanden sich 2 Geschwüre auf den grossen Lippen, ein Geschwür in der linken Genitocruralfalte, eine Menge Geschwüre im Introitus und um die Uretralpapille. Auf dem Collum uteri sah man eine hervorspringende Plaque mit rothem Rande und weissgelbem Belag sowie zahlreiche gefurchte Depressionen (,gleichwie die Oberfläche des Gehirns mit seinen Gyri und Sulci"). Ausserdem 2 Geschwüre in dem obersten Theil der Scheide. Positive Inoculation. Dauer: 3 Wochen.

2. 26jähriges Mädchen; 2 Geburten. Chronische Endometritis. Auf der grossen Lippe eine Menge folliculärer Geschwüre; anf dem Collum uteri 2 mareelonnirte, hervorspringende Plaques mit weissgrauem diphtheroidem Belag, bestehend aus mehreren durch Furchen getrennte Protuberanzen. Mehrere Geschwüre in der Scheide. Das Geschwür auf dem Collum heilte erst im Verlauf von 6 Wochen. Pat. hatte zugleich chronische Metritis und Endometritis und bei der Entlassung blieb eine Erosion auf dem Collum. Während 3 monatlicher Beobachtung kein Symptom von Syphilis.

Seit Molènes Mittheilung im Jahre 1881 scheint der diphtheroide Schanker auf dem Cervix (wie überhaupt das venerische Gebärmuttergeschwür) nur einmal in der Literatur erwähnt worden zu sein, und zwar von Di Bella \& Ingria (Sulla ulcera non infettante muliebre, La Sicilia medica. Tome I, ref. in diesem Archiv 1890), welche mittheilen, dass sie auf dem Collum renerische Geschwüre beobachtet haben, welchen in der Regel der scharfe Rand und der vertiefte Grund fehlte, während sie sich dagegen als eine graue, etwas über das Niveau der Schleimhaut hervorspringende, mit einer diphtheroïden Pseudomembran bedeckten Plaque zeigen. 
Wenn man auf Grund unserer und der in der Literatur berichteten Fälle eine Beschreibung des diphteroïden Schankers auf dem Collum geben soll, so lässt es sich in aller Kürze mit folgenden Worten thun. Das renerische Geschwür auf dem Collum uteri kann, anstatt sich als ein tiefes, scharfgerändertes Geschwür zu zeigen, als eine papulös hervorspringende, mit einer anhaftenden Pseudomembran bedeckte, zuweilen ganz geschwulstartige Masse mit unebener, gefurchter und knolliger Oberfläche, von gelber Farbe und mit polycyklischer oder runder Contour, welche von den Umgebungen scharf abgegrenzt ist, auftreten. Der erste und dritte von unsern 3 Fällen ist ein Typus für diese Form. Zu anderen Zeiten bildet dieses Geschwür, wie in unserem zweiten Falle, eine einzelne, nur schwach hervorspringende Plaque von der Grösse eines Markstücks, welche sonst dieselben Eigenthümlichkeiten wie im erstgenannten Falle darbietet. Beide Foraen zeigen grosse Neigung selbst bei leichter Berührung, z. B. mit einem Wattetampon, zu kleinen Hämorrhagien, eine Eigenthümlichkeit, welche in unsern Fällen sehr hervortretend war, und welche auch von früheren Beobachtern des weichen Schankers auf dem Collum im Allgemeinen erwähnt wird (Suchaneck, Scanzoni und Dawosky, cit. bei Schwartz). Die Frage, warum der Schanker in gewissen Fällen das beschriebene Aussehen annimmt, ist von den Verfassern, welche früher dahin gehörige Fälle veröffentlicht haben, nicht discutirt worden. Unsere drei Patienten hatten indessen alle eine rorherbestełende Gebärmutteraffection, welche uns wohl zu der Annahme berechtigt, dass sie den Boden auf dem Cervix so vorbereitet habe,' dass der Schanker sich auf so ungewöhnliche Weise hat entwickeln können. Alle Patienten batten nämlich eine chronische Endometritis mit den gewöhnlichen "Erosionen" auf dem Collum, und in beiden Fällen entsprach die Ausbreitung des Schankers ungefähr der Grösse dieser Affection. Auch in den verschiedenen in der Literatur mitgetheilten Fällen (wie in Molènes' beiden Fällen) findet man erwähnt, dass die Patienten, welche fäst alle geboren hatten, an Leukorrhöe oder chronischer Endometritis litten oder gelitten hatten, ohne dass man jedoch besonderes Gewicht auf diese Erscheinung legte. Es ist ein- 
leuchtend, dass die ron dem endometritischen Folgeleiden angegriffene Schleimhaut mit ihrem kranken und dünnen, an einzelnen Stellen fehlenden Epithelium die Fntwicklung des Schankers ganz anders begünstigen kann als ein gesundes Epithel. Von der Häufigkeit der hier beschriebenen Schankerform weiss man nicht viel, wahrscheinlich wird sie noch jetzt wie früher recht allgemein rerkannt. Ausser Molènes' 2 Fällen und Bernutz's 7 Fällen findet man in Schwartz' Thèse einzelne hierher gehörige Fälle. Fournier, welcher in den 6 Jahren, in denen er Oberarzt im Lourcine war, $25 \mathrm{Mal}$ das venerische Geschwïr auf dem Cervix beobachtet hat, gibt nicht an, wie oft dasselbe das papulöse, hypertrophische Aussehen gehabt habe, aber sagt in seinen Vorlesungen über Syphilis bei der Frau, dass es "häufig" diese Form annehme. Wenn man nach dem Studium aller Krankenberichte, welche iberhaupt über Schanker auf dem Cervix vorliegen, und in denen die Symptome und der Verlauf einigermassen sorgfältig beschrieben sind, urtheilt, so bekommt man den Eindruck, dass das renerische Geschwïr auf dem Cervix sich überhaupt weit häufiger in der hier beschriebenen Form als wie das gewöhnliche scharfgeränderte tiefe Geschwür zeigt. Diese Auffassung nähert sich auch der von Schwartz gegebenen Darstellung. Dieser Verfasser behauptet nämlich, dass Bernutz' diphtheroider Schanker keine Form sei, welche von den gewöhnlichen Formen scharf abgegrenzt sei, sondern dass sich alle möglichen Uebergänge zwischen den hypertrophischen diphtheroiden Schankern und den gewöhnlich beschriebenen Formen finden. Unser 3. Fall stützt diese Auffassung. Was die D a u er betrifft, so ist dieselbe weit grösser bei der diphtheroiden Form als bei einem Geschwür von der gewöhnlichen Form. Während dieses oft im Verlauf von einigen Tagen, selbst ohne irgend eine andere Behandlung als einfache Reinlichkeit, heilt, dauert die hier erwähnte Form viele Wochen, wenn keine Aetzung vorgenommen wird oder wenn nicht die Jodoformbehandlung eintritt. ${ }^{1}$ )

1) Die grosse Schnelligkeit (5-6 Tage), mit welcher das venerische Geschwür auf dem Uterus in der Regel heilt, wie die Thatsache, dass es nie von eiternden Bubonen in der Fossa iliaca begleitet ist, sowie endlich seine grosse Seltenbeit, wird leicht durch das von $A$ ubert be- 
Die Diagnose wird dem wahrscheinlich leicht sein, welcher diese Affection einmal gesehen oder nur von ihr gehört hat, da es keine andere Läsion gibt, welche dieser genau gleicht. Glaubt man die Diagnose nach dem Aussehen allein nicht stellen zu können, so werden fast immer an oder um die äusseren Geschlechtstheile ein oder mehrere venerische Geschwüre von dem gewöhnlichen Aussehen sein, so dass man durch sie leicht auf den rechten Weg geleitet wird. In den von Schwartz gesammelten 25 Fällen von Gebärmutterschankern waren $23 \mathrm{Mal}$ Schanker in der Vulva. Sollte man hier keine Schanker finden, muss man seine Zuflucht zu der Inoculation nehmen, welche beim positiven Resultat Sicherheit für die Diagnose gibt. Man muss hier darauf achten, dass man wirklich das Sekret von der Oberfläche des Schankers erhält, und man nicht von dem aus der Gebärmutterhöhle abgesonderten Eiter einimpft, welcher natirlich nur Inoculationsschanker geben wird, wenn das Geschwïr sich bis in den Cervicalcanal erstrecken möchte. Ebenfalls muss man sich natürlich hüten, die Inoculation zu einem Zeitpunkt vorzunehmen, wo kurz vorher mikrobicide Agentia mit der Oberfläche des Schankers in Berührung gewesen sind. Die Differentialdiagnose von dem syphilitischen Schanker wird zuweilen schwierig sein können; dieser hat aber, wie die selten vorkommenden Papeln auf dem Collum, eine mehr graue Farbe, welche recht verschieden von der hellgelben des weichen Schankers ist, und die Inoculation gibt natürlich ein negatives Resultat. Die Verhärtung, welche sonst, wenn es die Differentialdiagnose zwischen diesen beiden Krankheiten gilt, von so grosser Bedeutung ist, wird in der Regel schwierig, selbst bei typisch syphilitischen Schankern, wegen der Localität nachgewiesen werden können. Da die venerischen Geschwüre ausserdem oft von einer gewissen Entzündungsgeschwulst begleitet sein können, hat man bei den Schankern auf dem Collum wenig Vortheil von diesem Symptom. Zuweilen wird man genöthigt sein müssen, die Diagnose in suspenso zu lassen, bis sich vielleicht andere

wiesene Factum, dass das Agens des venerischen Geschwürs, wenn es 16-18 Stunden einer Temperatur von $37-38^{\circ}$ ausgesetzt ist, seine Virulens verliert. 
Symptome von Syphilis zeigen. Wenn das venerische Geschwür nur schwach erhaben, wie in unserm zweiten Fall, ist, wird die Diagnose besonders schwierig sein gegenüber den gewöhnlichen Erosionen, wie man sie bei Endometritis von gonorrhoischer oder anderer Natur findet, besonders wenn der Schanker zuweilen, wie in unserem Falle, mit einer solchen Affection zugleich auftritt. Hier spielt wieder die Farbe der Läsion eine Rolle. Die Erosionen haben in der Regel eine rothe Farbe, wogegen die Farbe des Schankers gelbroth, grauroth oder gelblich ist. Diese Farbe rührt von der anhaftenden diphtheroïden Pseudomembran, welche man nicht bei der Erosion findet, her. Man muss jedes Mal, wenn man auf einer Erosion auf dem Collum die beschriebene Pseudomembran findet, an die Möglichkeit eines weichen Schankers denken. Geben die äusseren Schanker keine Sicherheit für die Diagnose, so muss man sich diese durch Inoculation verschaffen. Endlich könute man sich denken, dass der diphtheroide Schanker in seiner hyperplastischen Form, wie in unserem ersten Fall, einem Beobachter, welcher denselben zum ersten Mal sieht, Veranlassung zur Verwechselung mit einem Epitheliom geben könnte. Fine Excision mit folgender mikroskopischer Untersuchung wird diese Diagnose bald ausschliessen.

Die Behandlung muss dieselbe sein wie bei dem venerischen Geschwür im allgemeinen. Das von Ern e s t B e s n i er im Jahre 1867 in die Therapie dieser Krankheit eingeführte Jodoform feiert hier grosse Triumphe. In unserem ersten Falle war es überraschend zu sehen, welche Veränderung mit der Affection bei einer nur 3-4tägigen Jodoformbehandlung vor sich ging. Ausserdem muss man natürlich durch häufige Scheidendouchen für eine peinliche Reinlichkeit sorgen, um den Pat., soweit wie möglich, vor Antoinoculationen zu bewahren. 